\title{
Tangent map intermittency as an approximate analysis of intermittency in a high dimensional fully stochastic dynamical system: The Tangled Nature model
}

Alvaro Diaz-Ruelas, Henrik Jeldtoft Jensen, Duccio Piovani, and Alberto Robledo

Citation: Chaos 26, 123105 (2016); doi: 10.1063/1.4968207

View online: https://doi.org/10.1063/1.4968207

View Table of Contents: http://aip.scitation.org/toc/cha/26/12

Published by the American Institute of Physics

\section{Articles you may be interested in}

Counting forbidden patterns in irregularly sampled time series. I. The effects of under-sampling, random depletion, and timing jitter

Chaos: An Interdisciplinary Journal of Nonlinear Science 26, 123103 (2016); 10.1063/1.4968551

Method for reconstructing nonlinear modes with adaptive structure from multidimensional data

Chaos: An Interdisciplinary Journal of Nonlinear Science 26, 123101 (2016); 10.1063/1.4968852

Local noise sensitivity: Insight into the noise effect on chaotic dynamics

Chaos: An Interdisciplinary Journal of Nonlinear Science 26, 123102 (2016); 10.1063/1.4970322

Counting forbidden patterns in irregularly sampled time series. II. Reliability in the presence of highly irregular sampling

Chaos: An Interdisciplinary Journal of Nonlinear Science 26, 123104 (2016); 10.1063/1.4970483

On the estimation of phase synchronization, spurious synchronization and filtering

Chaos: An Interdisciplinary Journal of Nonlinear Science 26, 123106 (2016); 10.1063/1.4970522

Dynamics of bow-tie shaped bursting: Forced pendulum with dynamic feedback

Chaos: An Interdisciplinary Journal of Nonlinear Science 26, 123107 (2016); 10.1063/1.4971411

\section{Don't let your writing keep you from getting published!}




\title{
Tangent map intermittency as an approximate analysis of intermittency in a high dimensional fully stochastic dynamical system: The Tangled Nature model
}

\author{
Alvaro Diaz-Ruelas, ${ }^{1, \text { a) }}$ Henrik Jeldtoft Jensen, ${ }^{2, b)}$ Duccio Piovani, ${ }^{2, c)}$ \\ and Alberto Robledo ${ }^{3, d)}$ \\ ${ }^{1}$ Instituto de Física, Universidad Nacional Autónoma de México, Ciudad Universitaria, Ciudad de México \\ 04510, Mexico \\ ${ }^{2}$ Centre for Complexity Science and Department of Mathematics, Imperial College London, South Kensington \\ Campus, London SW7 2AZ, United Kingdom \\ ${ }^{3}$ Instituto de Física y Centro de Ciencias de la Complejidad, Universidad Nacional Autónoma de México, \\ Ciudad Universitaria, Ciudad de México 04510, Mexico
}

(Received 1 April 2016; accepted 8 November 2016; published online 6 December 2016)

\begin{abstract}
It is well known that low-dimensional nonlinear deterministic maps close to a tangent bifurcation exhibit intermittency and this circumstance has been exploited, e.g., by Procaccia and Schuster [Phys. Rev. A 28, 1210 (1983)], to develop a general theory of $1 / f$ spectra. This suggests it is interesting to study the extent to which the behavior of a high-dimensional stochastic system can be described by such tangent maps. The Tangled Nature (TaNa) Model of evolutionary ecology is an ideal candidate for such a study, a significant model as it is capable of reproducing a broad range of the phenomenology of macroevolution and ecosystems. The TaNa model exhibits strong intermittency reminiscent of punctuated equilibrium and, like the fossil record of mass extinction, the intermittency in the model is found to be non-stationary, a feature typical of many complex systems. We derive a mean-field version for the evolution of the likelihood function controlling the reproduction of species and find a local map close to tangency. This mean-field map, by our own local approximation, is able to describe qualitatively only one episode of the intermittent dynamics of the full TaNa model. To complement this result, we construct a complete nonlinear dynamical system model consisting of successive tangent bifurcations that generates time evolution patterns resembling those of the full TaNa model in macroscopic scales. The switch from one tangent bifurcation to the next in the sequences produced in this model is stochastic in nature, based on criteria obtained from the local mean-field approximation, and capable of imitating the changing set of types of species and total population in the TaNa model. The model combines full deterministic dynamics with instantaneous parameter random jumps at stochastically drawn times. In spite of the limitations of our approach, which entails a drastic collapse of degrees of freedom, the description of a high-dimensional model system in terms of a low-dimensional one appears to be illuminating. Published by AIP Publishing. [http://dx.doi.org/10.1063/1.4968207]
\end{abstract}

Intermittent dynamics in the form of long periods of little change separated by relatively short time intervals of hectic activity is observed in many complex systems. Examples include snoring, mass extinctions, financial crashes, and brain activity. Such systems contain large numbers of components and very often involve stochastic processes. It was more than 40 years ago suggested by Procaccia and Schuster that important aspects of such intermittent dynamics can be captured by a simple essentially deterministic equation. It is not in general straightforward to connect in detail the complex system to the mathematics considered by Procaccia and Schuster. We consider a multi-component model of evolutionary ecology and derive how the single component equation of the

\footnotetext{
a)alvaropdr@fisica.unam.mx.

b) h.jensen@imperial.ac.uk.

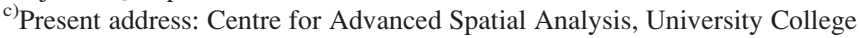
London, W1T 4TJ London, United Kingdom. Electronic mail: duccio.piovani@gmail.com.

d)robledo@fisica.unam.mx.
}

type considered by Procaccia and Schuster is related to the parameters of the stochastic many component dynamics. The single component description enables us to describe aspects of the intermittent extinction dynamics that so far has eluded mathematical analysis. We also demonstrate that the single component mathematics is able to qualitatively mimic the evolution and extinction dynamics of consecutive ecologies generated by the full many component model. We think that our results expands the applicability of the analysis put forward by Procaccia and Schuster and thereby help connect the methodology developed for deterministic and typically low dimensional dynamics to the stochastic dynamics of complex systems.

\section{INTRODUCTION}

High-dimensional complex systems, such as turbulence, relaxing glasses, biological evolution, the financial market or 
brain dynamics, exhibit intermittent dynamics. ${ }^{1,2}$ While intermittency in basic one-dimensional non-linear maps at the socalled tangent bifurcation ${ }^{2}$ has received significant attention, e.g., because of their universal aspects ${ }^{3}$ and has been suggested as a universal mechanism for $1 / f$ noise. ${ }^{4}$ In fact, the paradigmatic Pomeau-Manneville map ${ }^{5}$ was derived to represent intermittency in weakly turbulent fluid dynamics. The relevance of such maps to high-dimensional stochastic systems depends on whether a robust macroscopic degree of freedom emerges, which is able to capture the dominant dynamics.

A case in point is the Tangled Nature (TaNa) model ${ }^{1}$ of evolutionary ecology, since it displays intermittent evolution at the macroscopic level while microscopically individuals reproduce, mutate, and die at essentially constant rates. ${ }^{6,7}$ Numerical simulations of the model show that the total population $N(t)$ as a function of time $t$ (in the scale of generations) consists of quasi-stable, steady, periods that alternate with interludes of hectic transitions, during which $N(t)$ exhibits large amplitude fluctuations. ${ }^{6,7}$ The populations of species behave accordingly, during the quasi-stable periods they predominantly retain their identity, but at the transitions some species vanish, others arise, while the rest survive. ${ }^{6,7}$

Here, we study the incidence of intermittency, as displayed close to the tangent bifurcation in low-dimensional nonlinear maps, in the macroscopic behavior of the $\mathrm{TaNa}$ model. We make two intents. The first one is to approximate the evolution equations of the model, via determination of mean-field lowest-order local terms to obtain a map near tangency that reproduces the prototypical quasi-stable episode. The second is to model, phenomenologically, the sequences of consecutive quasi-stable and hectic periods via a nonlinear dynamical model that makes use of the families of tangent bifurcations that occur in one-dimensional quadratic maps. We arrive at the following general picture. The dynamics of the original TaNa model is fully stochastic, and fluctuations are very important in steering its progress. The one-dimensional mean-field local map near tangency, we derive, is deterministic and has the limitation, without further development, that only one quasi-stable event can be generated at a time. But this map offers criteria for the duration of the quasi-stable episodes in terms of the TaNa model parameters. Based on these criteria, we introduce a stochastic element in the construction of an otherwise deterministic nonlinear dynamical model. This model reproduces qualitatively the sequences of quasi-stable periods with varying types and numbers of species as in the TaNa model. We comment on the class of nonlinear dynamical systems with this kind of stochastic element. We reach the conclusion that in spite of the approximations incurred and assumptions made, our study facilitates interesting insights that hint to a radical reduction of degrees of freedom under certain circumstances.

\section{THE TANGLED NATURE MODEL}

The Tangled Nature model is a model of evolutionary ecology, which studies the macro-dynamics emerging from the dynamics of individual organisms or agents, co-evolving together and subject to a web of mutual interactions. The model is an attempt to identify possible simple mechanisms behind the myriad of complicated interactions, feedback loops, contingencies, etc., as one moves from the short time reproductive dynamics at the level of individuals to the long time system level behaviour. The strategy is to keep the model sufficiently simple to enable analysis, and to pinpoint the details or assumptions in the model that are responsible for the specific behaviour at the systems level. One major concern of the model has been to understand how the smooth continuous pace of the reproductive dynamics at the level of individuals can lead to intermittent or punctuated dynamics at the level of high taxonomic structures. The model was introduced in Refs. 6 and 7, and since then, the model framework has been used by several authors (see, e.g., Refs. $8-12$ ). A summary of some of the models features and predictions can be found in Ref. 1 .

\section{Description of the model}

The dynamical entities of the TaNa model consist of agents represented by a sequence of binary variables with fixed length $L .{ }^{13}$ We denote by $n\left(\mathbf{S}^{a}, t\right)$ the number of agents of type $\mathbf{S}^{a}=\left(S_{1}^{a}, S_{2}^{a}, \ldots, S_{L}^{a}\right)$ (here, $S_{i}^{a} \in\{-1,1\}$ ) at time $t$ and the total population is $N(t)=\sum_{a=1}^{2^{L}} n\left(\mathbf{S}^{a}, t\right)$. A time step is defined as a succession of one annihilation and of one reproduction attempt. Annihilation consists of choosing an agent at random with uniform probability and removing the agent with probability $p_{\text {kill }}$, taken to be constant in time and independent on the type. Reproduction: choose with uniform probability an agent, $\mathbf{S}^{a}$, at random and duplicate the agent (and remove the mother) with probability

$$
p_{k i l l}\left(\mathbf{S}^{a}, t\right)=\frac{\exp \left(H\left(\mathbf{S}^{a}, t\right)\right)}{1+\exp \left(H\left(\mathbf{S}^{a}, t\right)\right)},
$$

which depends on the occupancy distribution of all the types at time $t$ through the weight function

$$
H\left(\mathbf{S}^{a}, t\right)=\frac{k}{N(t)} \sum_{b} J\left(\mathbf{S}^{a}, \mathbf{S}^{b}\right) n\left(\mathbf{S}^{b}, t\right)-\mu N(t) .
$$

In Eq. (2), the first term couples the agent $\mathbf{S}^{a}$ to one of type $\mathbf{S}^{b}$ by introducing the interaction strength $\mathbf{J}\left(\mathbf{S}^{a}, \mathbf{S}^{b}\right)$, whose values are randomly distributed in the interval $[-1,+1]$. For simplification and to emphasize interactions, we here assume: $\mathbf{J}\left(\mathbf{S}^{a}, \mathbf{S}^{a}\right)=0$. The parameter $k$ scales the interaction strength and $\mu$ can be thought of as the carrying capacity of the environment. An increase (decrease) in $\mu$ corresponds to harsher (more favourable) external conditions.

Mutations occur in the following way: For each of the two copies $\mathbf{S}^{a_{1}}$ and $\mathbf{S}^{a_{2}}$, a single mutation changes the sign of one of the genes: $S_{i}^{a_{1}} \rightarrow-S_{i}^{a_{1}}, S_{i}^{a_{2}} \rightarrow-S_{i}^{a_{2}}$ with probability $p_{\text {mut }}$. We define a generation to consist of $N(t) / p_{\text {kill }}$ time steps, i.e., the average time needed to kill all the individuals at time $t$. These microscopic rules generate intermittent macrodynamics ${ }^{7}$ as shown in Fig. 1. The long quiescent epochs are called quasi Evolutionary Stable Strategies (qESS), since they do remind one of John Maynard Smith's notion of 

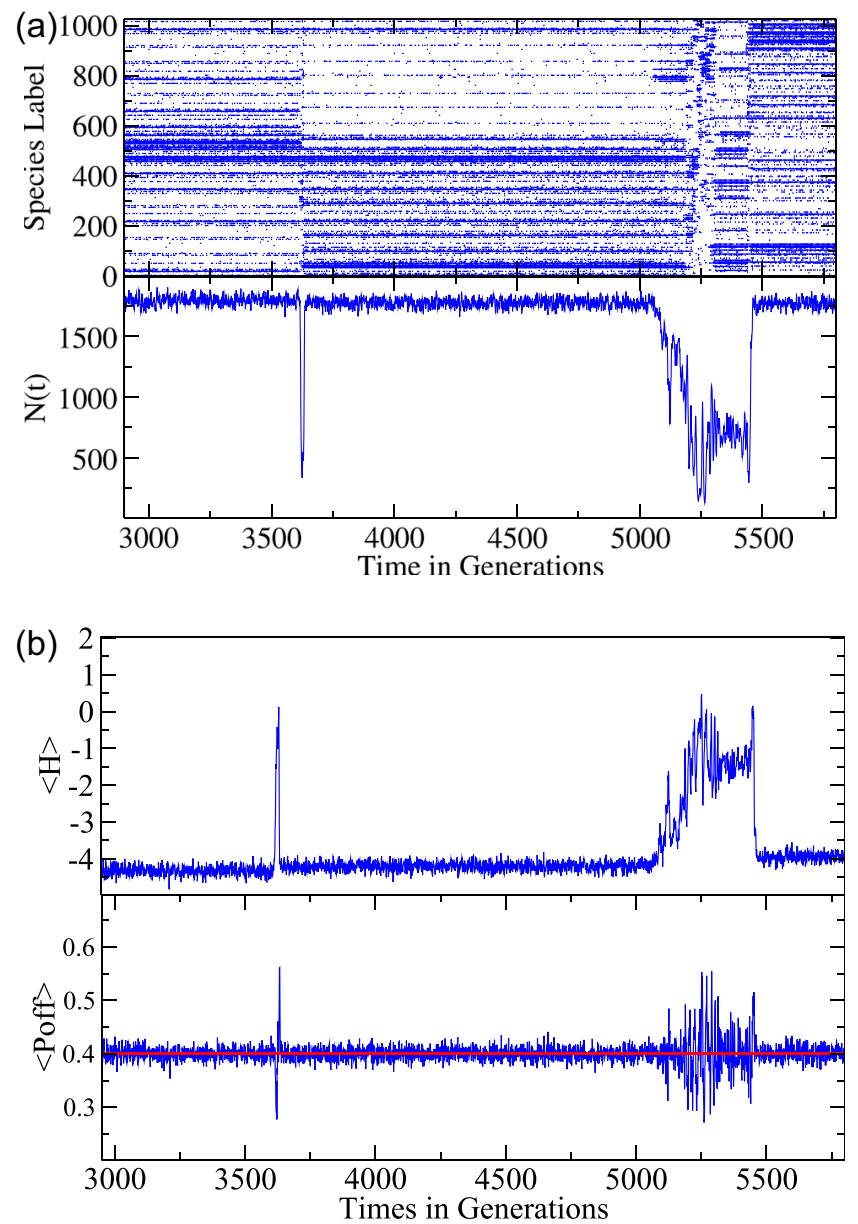

FIG. 1. Left panel: Total population as a function of time (in generations) for a single realization of the TaNa model. The punctuated dynamics is clearly visible: quasi-stable periods alternate with periods of hectic transitions, during which $N(t)$ exhibits large amplitude fluctuations. Right panel: The average of the weight function $H$ and the reproduction probability. The parameters are $L=10, p_{\text {kill }}=0.4, p_{\text {mut }}=0.02, \mu=0.007, k=40$ the red line indicates $p_{\text {kill }}$.

Evolutionary Stable Strategies introduced in his game theoretic description of evolution. ${ }^{14}$

The weight function $H$ will fluctuate about the value given by the stable dynamical fixed point condition $p_{\text {off }}(H)=p_{\text {kill }}$. This suggests that the mean field value of $H$ may indeed evolve in an intermittent way that may be captured by a tangent map. We will therefore derive the mean field map for $\langle H\rangle$.

\section{Derivation of mean field map for $\boldsymbol{H}$}

To establish a map for the mean field approximation to the weight function $H$, we need to analyse each of the microscopic stochastic processes that can lead to a change in $H$. These are reproduction, with or without mutation and death. And we will make use of the fact that if a quantity, say, $X$, undergoes the change to $X \mapsto X+\Delta$ with probability $p$ and remains unchanged $X \mapsto X$ with probability $1-p$, then in the mean field approximation, we have $\langle X\rangle \mapsto\langle X\rangle+p \Delta$. We use a short hand notation in which we label individuals and types as $i, j, k \ldots$ and accordingly the interaction between two types $i$ and $j$ as $J_{i j}$.
Reproduction with no mutation: We need to estimate the average change to the likelihood function $H_{i}$ for type $i$, given that an individual of type $j_{0}$ reproduces without mutating. The change in $H_{i}$ is given by

$$
\begin{aligned}
H_{i} & \mapsto \frac{k}{N+1}\left[\sum_{j \neq j_{0}} J_{i, j} n_{j}+J_{i, j_{0}}\left(n_{j_{0}}+1\right)\right]-\mu(N+1) \\
& =\frac{k}{N+1}\left(\sum_{j} J_{i, j} n_{j}-\mu N\right)+\left(\frac{k}{N+1} J_{i, j_{0}}-\mu\right) \\
& =H_{i}+\Delta_{R, 0 m}^{i}\left(j_{0}\right) .
\end{aligned}
$$

We replaced $N+1$ by $N$ in the first term and introduced the change

$$
\Delta_{R, 0 m}^{i}\left(j_{0}\right)=\frac{k}{N+1} J_{i, j_{0}}-\mu
$$

which will occur with probability

$$
p_{R, 0 m}^{i}\left(j_{0}\right)=\frac{n_{j_{0}}}{N} p_{o f f}\left(j_{0}\right)\left(P_{m u t}^{(0)}\right)^{2},
$$

where $P_{m u t}^{(0)}=\left(1-p_{m u t}\right)^{L}$ is the probability of no mutations occurring, and its counted twice, once for each offspring. Averaging over all possible types (of which there are $\Omega=2^{L}$ ), we obtain

$$
\begin{aligned}
\bar{\Delta}_{R, 0 m} & =\left\langle\Delta_{R, 0 m}^{i}\left(j_{0}\right)\right\rangle=\frac{1}{\Omega} \sum_{j_{0}}\left(\frac{k}{N} J_{i j_{0}}-\mu\right) \frac{n_{j_{0}}}{N} p_{\text {off }}\left(j_{0}\right)\left(P_{m u t}^{(0)}\right)^{2 L} \\
& \mapsto\left(\frac{k \bar{J}}{N}-\mu\right)\left\langle p_{\text {off }}\right\rangle_{\text {ext }}\left(1-p_{\text {mut }}\right)^{2 L}
\end{aligned}
$$

where we have introduced $\bar{J}$, which denotes the strengths $J_{i, j}$ averaged over pairs of interacting extant types and similarly $\left\langle p_{\text {off }}\right\rangle_{\text {ext }}$ denotes the offspring probability average over extant types.

Reproduction with 1 mutation: Next, we consider the average change to the likelihood function, $H_{i}$ for type $i$ given that an individual of type $j_{0}$ reproduces with one copy mutating and ending in $q_{o}$ and the other not mutating. The change in $H_{i}$ is given by

$$
\begin{aligned}
H_{i} & \mapsto \frac{k}{N+1}\left[\sum_{j \neq q_{0}} J_{i, j} n_{j}+J_{i, q_{0}}\left(n_{q_{0}}+1\right)\right]-\mu(N+1) \\
& =\frac{k}{N+1}\left(\sum_{j} J_{i, j} n_{j}-\mu N\right)+\left(\frac{k}{N+1} J_{i, q_{0}}-\mu\right) \\
& =H_{i}+\Delta_{R, m}^{i}\left(q_{0}\right) .
\end{aligned}
$$

Again, we have replaced $N+1$ by $N$ in the first term and introduced the change

$$
\Delta_{R, m}^{i}\left(q_{0}\right)=\frac{k}{N+1} J_{i, q_{0}}-\mu
$$

which will occur with probability

$$
p_{R, m}^{i}\left(j_{0}\right)=\frac{n_{j_{0}}}{N} p_{o f f}\left(j_{0}\right) p_{j_{o} \rightarrow q_{o}}
$$


where

$$
p_{j_{o} \rightarrow q_{o}}=p_{\text {mut }}^{d_{j o q_{o}}}\left(1-p_{\text {mut }}\right)^{L-d_{j o q_{o}}}
$$

and $d_{j_{o} q_{o}}$ is the hamming distance between the sequences $j_{o}$ and $q_{o}$. This means that

$$
\bar{\Delta}_{R, 1 m}^{i}=\sum_{j_{o} q_{o}}\left(\frac{k}{N+1} J_{i, q_{o}}-\mu\right) \frac{n_{j_{o}}}{N} p_{j}^{o f f} p_{m u t}^{d_{j o q_{o}}}\left(1-p_{m u t}\right)^{L-d_{j o q_{o}}}
$$

By limiting our approximation to the nearest neighbours, and proceeding like in the previous case, we obtain

$$
\bar{\Delta}_{R, 1 m}=L p_{m u t}^{(o)} p_{m u t}^{(1)}\left(\frac{k \tilde{J}}{N}-\mu\right)\left\langle p_{\text {off }}\right\rangle_{\text {ext }},
$$

where $L$ is the number of first neighbours and $P_{m u t}^{(1)}=p_{\text {mut }}$ $\left(1-p_{\text {mut }}\right)^{(L-1)}$ denotes the probability that exactly one $L$ genes mutate. Notice the difference between $\bar{J}$ introduced in Eq. (6) and the averaged quantity $\tilde{J}$ introduced in this equation. The two differs by being averages over different sets of types. Here, $\tilde{J}$ is averaged over interaction strengths $J_{i j}$ connecting already occupied type and types hit by a new mutation, i.e., types located in the perimeter of the cluster of extant reproducing sites. In contrast, $\bar{J}$ is the average of the interaction strength between extant types. We will expect that typically $\tilde{J}<\bar{J}$ because adaptation has favoured mutualistic interactions amongst the extant types. However, an accurate estimate of the two quantities from first principle is of course very difficult.

Reproduction with 2 mutations: Next, we consider the average change to the likelihood function $H_{i}$ for type $i$, given that an individual of type $j_{0}$ reproduces with both copies mutating and ending in $q_{o}$ and $q_{1}$. The change in $H_{i}$ is given by

$$
\begin{aligned}
H_{i} \mapsto & \frac{k}{N+1}\left[\sum_{j \neq q_{0}, q_{1}, j_{o}} J_{i, j} n_{j}+J_{i, q_{0}}\left(n_{q_{0}}+1\right)\right. \\
& \left.+J_{i, q_{1}}\left(n_{q_{1}}+1\right)+J_{i, q_{1}}\left(n_{q_{1}}-1\right)\right]-\mu(N+1) \\
= & \frac{k}{N+1}\left(\sum_{j} J_{i, j} n_{j}-\mu N\right) \\
& +\left(\frac{k}{N+1}\left(J_{i, q_{0}}+J_{i, q_{1}}-J_{i, j_{o}}\right)-\mu\right) \\
= & H_{i}+\Delta_{R, 2 m}^{i}\left(q_{0}\right),
\end{aligned}
$$

where we have considered the fact that the number of individuals of the parent decreases in the case of 2 mutations. Again, we have replaced $N+1$ by $N$ in the first term and introduced the change

$$
\Delta_{R, 2 m}^{i}\left(q_{0}\right)=\frac{k}{N+1}\left(J_{i, q_{0}}+J_{i, q_{1}}-J_{i, j_{o}}\right)-\mu,
$$

which will occur with probability

$$
p_{R, m}^{i}\left(j_{0}\right)=\frac{n_{j_{0}}}{N} p_{o f f}\left(j_{0}\right) p_{j_{o} \rightarrow q_{o}} p_{j_{o} \rightarrow q_{1}} .
$$

And once again limiting our approximation to the nearest neighbour mutations, we obtain

$$
\bar{\Delta}_{R, 2 m}=L^{2}\left(P_{m u t}^{(1)}\right)^{2}\left(\frac{k \tilde{J}}{N}-\mu\right)\left\langle p_{\text {off }}\right\rangle_{\text {ext }} .
$$

Notice the difference between $\bar{J}$ introduced in Eq. (6) and the averaged quantity $\tilde{J}$ introduced in this equation. The two differs by being averages over different sets of types. Here, $\tilde{J}$ is averaged over interaction strengths $J_{i j}$ connecting already occupied type and types hit by a new mutation, i.e., types located in the perimeter of the cluster of extant reproducing sites. In contrast $\bar{J}$ is the average of the interaction strength between extant types. We will expect that typically $\tilde{J}<\bar{J}$ because adaptation has favoured mutualistic interactions amongst the extant types. This is in fact verified by our simulations (see Fig. 3).

Killing event on site $j_{0}$ leads to

$$
\begin{aligned}
H_{i} & \mapsto \frac{k}{N-1}\left[\sum_{j \neq j_{0}} J_{i, j} n_{j}+J_{i, 0_{0}}\left(n_{j_{0}}-1\right)\right]-\mu(N-1) \\
& =\frac{k}{N-1}\left(\sum_{j} J_{i, j} n_{j}-\mu N\right)-\left(\frac{k}{N} J_{i, j_{0}}-\mu\right) \\
& =H_{i}-\Delta_{R, 0 m}^{i}\left(j_{0}\right) .
\end{aligned}
$$

This change occurs with probability $\left(n_{j_{0}} / N\right) p_{\text {kill }}$.

Combining this result with the weighted results in Eqs. (6), (12), and (16), we obtain the following map, in which the mean field describes how $\langle H\rangle$ changes as an effect of the microscopic reproduction and killing events

$$
\langle H\rangle \mapsto\langle H\rangle+A\left\langle p_{\text {off }}\right\rangle_{\text {ext }}-B p_{\text {kill }},
$$

where the coefficients are given by

$$
\begin{gathered}
A=\left(\frac{k \bar{J}}{N}-\mu\right)\left(P_{m u t}^{(0)}\right)^{2}+\left(\frac{k \tilde{J}}{N}-\mu\right)\left(P_{m u t}^{(0)}+L p_{m u t}^{(1)}\right) L p_{m u t}^{(1)}, \\
B=\frac{k \bar{J}}{N}-\mu .
\end{gathered}
$$

We have derived a map for the evolution of $\langle H\rangle$. We now need to close the map, i.e., we need a way to express the $H^{i}$ dependency of $\left\langle p_{\text {off }}\right\rangle_{\text {ext }}$ in terms of $\langle H\rangle$. We could assume

$$
\left\langle p_{\text {off }}\left(H^{i}\right)\right\rangle_{\text {ext }} \mapsto p_{\text {off }}\left(\langle H\rangle_{\text {ext }}\right)
$$

This procedure gives us the following map for $x_{n}$ (which we use as shorthand for the iterates of $\left.\langle H\rangle_{\text {ext }}\right)$ :

$$
x_{n+1}=x_{n}+A p_{o f f}\left(x_{n}\right)-B p_{k i l l} .
$$

The map has a fixed point a $x^{*}$ given by $p_{\text {off }}\left(x^{*}\right)=B p_{\text {kill }} / A$. The map is stable if $A<0$ and $B<0$. For $A B<0$, the map is either attractive (repulsive) to the left of $x^{*}$ and repulsive (attractive) to the right hand of $x^{*}$. For $A<0$ and $B<0, x^{*}$ is 
repulsive in both directions. The conclusion is that the dramatic mean field approximation suggested in Eq. (21), which corresponds to the replacement $\left\langle H^{n}\right\rangle_{\text {ext }} \mapsto\langle H\rangle_{\text {ext }}^{n}$ for all $n \in \mathbb{N}$, wipes out the intermittency. To establish a mean field description of the intermittency, we instead expand $p_{\text {off }}\left(H^{i}\right)$ in Eq. (18) to second order about $x^{*}$ and replaces only $\left\langle H^{2}\right\rangle_{\text {ext }}$ by $\langle H\rangle_{\text {ext }}^{2}$. This leads to a tangent map and we study the intermittency of this map in section: A CONSECUTIVE TANGENT BIFURCATION MODEL.

\section{ANALYSIS OF THE MAP IN THE NEIGHBOURHOOD OF TANGENCY}

We expand $p_{\text {off }}(H)$ in Eq. (18) to second order about $H^{*}=\ln \left[p_{\text {kill }} /\left(1-p_{\text {kill }}\right)\right]$

$$
p_{o f f}(H)=a_{0}+a_{1}\left(H-H^{*}\right)+a_{2}\left(H-H^{*}\right)^{2},
$$

where

$$
\begin{aligned}
& a_{0}=p_{\text {kill }}, \\
& a_{1}=p_{o f f}^{\prime}\left(H^{*}\right)=p_{\text {kill }}\left(1-p_{\text {kill }}\right), \\
& a_{2}=\frac{1}{2} p_{\text {off }}^{\prime \prime}\left(H^{*}\right)=\frac{1}{2} a_{1}\left(1-2 p_{\text {kill }}\right) .
\end{aligned}
$$

We substitute Eq. (23) into Eq. (22) and obtain the following map for $\Delta=\langle H\rangle-H^{*}$ :

$$
\Delta_{n+1}=b_{0}+b_{1} \Delta_{n}+b_{2} \Delta_{n}^{2} \equiv f\left(\Delta_{n}\right) \text {, }
$$

where

$$
\begin{aligned}
& b_{0}=a_{0}(A-B), \\
& b_{1}=1+a_{1} A, \\
& b_{2}=a_{2} A .
\end{aligned}
$$

Let $\Delta_{c}$ be given by $f^{\prime}\left(\Delta_{c}\right)=1$ and $\epsilon=f\left(\Delta_{c}\right)-\Delta_{c}$, i.e., at $\Delta_{c}$, the map has a tangent parallel to the identity and the vertical distance to the identity at this point is $\epsilon$ and is given by

$$
\epsilon=b_{0}-\frac{\left(1-b_{1}\right)^{2}}{4 b_{2}}
$$

In Figure 2, we show an example of an iteration of the map in Eq. (24) for a set of typical simulation parameters.

The number of iterations $T$ needed to pass through the bottleneck between the map and the identity is of the order $T=\pi / \sqrt{\epsilon b_{2}}$ (see, e.g., Ref. 2, Chap. 5). Hence, we have

$$
\left(\frac{\pi}{T}\right)^{2}=b_{0} b_{2}-\frac{1}{4}\left(1-b_{1}\right)^{2}
$$

We can simplify this expression by only working to the lowest order in the killing probability, and furthermore, we will only include mutation processes considered above, i.e., single gene mutations in one or in both offspring. Let us denote by $P_{0}$ the probability that no mutation occur, i.e., $P_{0}=\left(1-p_{\text {mut }}\right)^{2 L}$. Since we neglect all other mutation events than the two kinds just described, we have the approximation

$$
1-P_{0}=\left(P_{m u t}^{(0)}+L p_{m u t}^{(1)}\right) L p_{m u t}^{(1)},
$$

in which case Eq. (19) becomes

$$
A=\left(\frac{k \bar{J}}{N}-\mu\right) P_{0}+\left(\frac{k \tilde{J}}{N}-\mu\right)\left(1-P_{0}\right) .
$$

With these approximations, we arrive at

$$
\left(\frac{\pi}{T}\right)^{2} \simeq-\frac{k}{2 N}(\bar{J}-\tilde{J})\left(1-P_{0}\right)\left[\frac{k}{N}(\bar{J}-\tilde{J}) P_{0}+\frac{k}{N} \tilde{J}-\mu\right] p_{k i l l}^{2} .
$$

We find that $k P_{0}(\bar{J}-\tilde{J}) / N$ is very small and hence that the expression for $(\pi / T)^{2}$ is well approximated by

$$
\left(\frac{\pi}{T}\right)^{2} \simeq-\frac{k}{2 N}(\bar{J}-\tilde{J})\left(1-P_{0}\right)\left(\frac{k}{N} \tilde{J}-\mu\right) p_{k i l l}^{2} .
$$

We conclude that our mean field analysis suggests that the length of the qESS, i.e., the metastable quiescent epochs, is set by four mechanisms. First, it is obvious that the rate of mutations $\left(1-P_{0}\right)$ influences the duration of the qESS: no mutations leads to no transitions and hence lead to $T=\infty$. Second, the rate of killing, i.e., the factor $p_{\text {kill }}^{2}$. The rate of killing is related to the rate of offspring production, since due to the environmental coupling term $-\mu N$ in Eq. (2) on
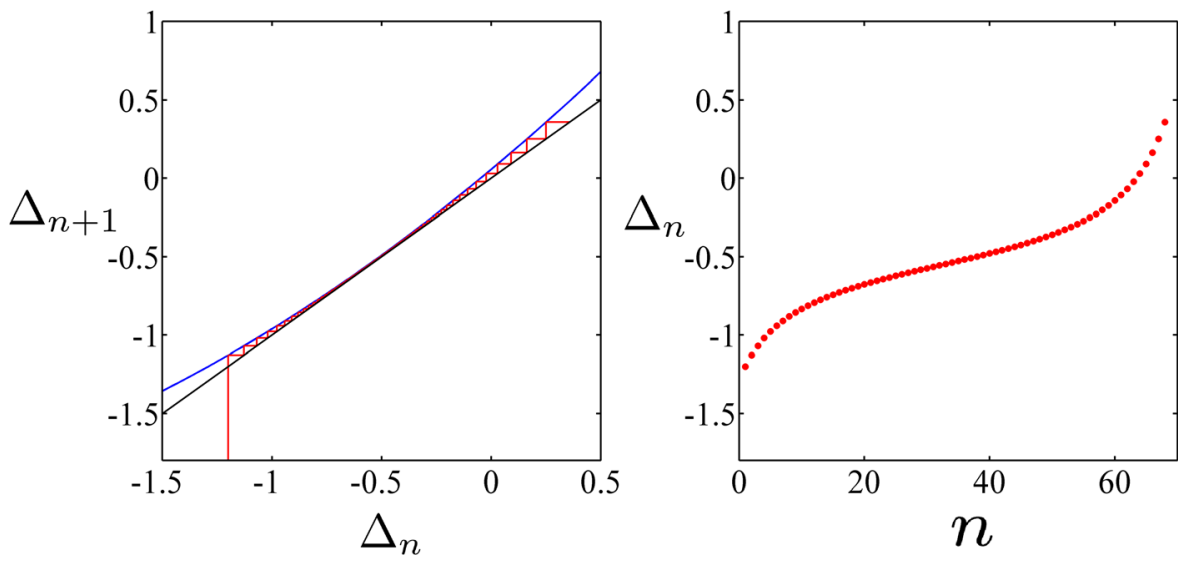

FIG. 2. The left panel shows the first 67 iterations of the map in Eq. (24), with initial condition $\Delta_{0}=-1.2$. The corresponding trajectory is shown in the right panel. The set of parameter values is the same than that as for Fig. 1, with the corresponding averaged interactions $\bar{J} \approx 0.0587$ and $\tilde{J} \approx-0.000001$, thus yielding the coefficients of the map $b_{0} \approx 0.060784, b_{1} \approx 1.167990, \quad$ and $b_{2} \approx 0.151191$. 
average there is a balance between reproduction and killing. Hence, if $p_{\text {kill }}$ decreases the rate of reproduction decreases and fewer mutations are produced leaving the qESS more stable and $T$ larger. The third mechanism influencing $T$ is the mismatch between the characteristic interaction strength between the set of extant types and mutant types located in the perimeter of the set of extant types, i.e., the factor $(\bar{J}-\tilde{J})$. One may consider this factor as being related to the selective pressure on the qESS state. Namely, if $\bar{J}=\tilde{J}$, there is no selective pressure, since the mutants are entirely like the wild types. The lack of selective pressure drives $T$ to become infinite. Finally, the term $k \tilde{J}-\mu$. Again one may see the fact that when $k \tilde{J} / N=\mu$ the duration $T$ becomes infinite as representing the fact that a mutant population for which $\tilde{k} / N=\mu$ is already well tuned to the environmental pressure represented by $\mu$.

It is of course interesting to try to relate the prediction for the duration $T$ given by Eq. (30) to the actual qESS intermittency observed in simulations of the Tangled Nature model. To do this, we have determined the time average values of $\bar{J}$ and $\tilde{J}$ during 4000 qESS states and plot the distributions obtained in Fig. 3. In order for Eq. (30) to make sense the sign has of course to be positive. The two terms that could in principle be problematic are $(\bar{J}-\tilde{J})$ and $\left(\frac{k \tilde{J}}{N}-\mu\right)$. In Fig. 3, we show the distribution of these terms obtained from a set of 4000 measured qESS. The difference in the average $J$ 's is always positive while the second term is always negative. Given the high stochasticity of the TaNa, this does not mean that a specific qESS cannot produce a negative value, but that this is extremely unlikely, and perhaps it would not last long enough for us to notice it. Amongst all the realisations of $\bar{J}$ and $\tilde{J}$ we recorded, only $0.16 \%$ corresponds to a negative right hand side in Eq. (30).

Indeed one has to consider Eq. (30) as a qualitative description of the duration more than a quantitative one. This is the case because of the approximations we were forced to make when deriving the mean field expressions. Moreover, it is difficult to relate the quantitative values for $T$ obtained from Eq. (30) to the durations observed in the simulations because of the always difficult problem of relating mean field time to Monte Carlo simulation time steps.

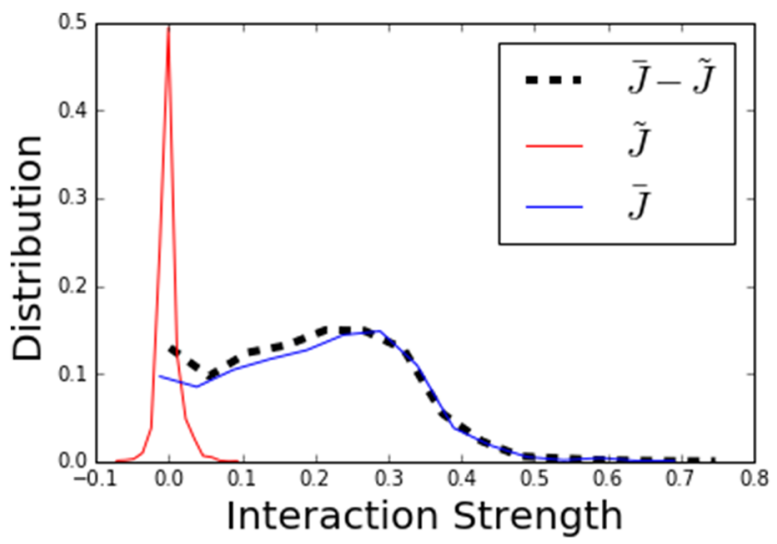

\section{A CONSECUTIVE TANGENT BIFURCATION MODEL}

Based on the analysis in Derivation of a Mean field map for $\mathrm{H}$, we now advance a simple nonlinear dynamical model capable of imitating some features of the macroscopic dynamics that can be typically generated by the TaNa model.

It is important to notice that the local tangent map we obtained in Eq. (2) can only generate one qESS episode since it does not provide a reinjection mechanism or alternative continuation of dynamical evolution. Therefore, to reproduce sequences of qESS episodes it is necessary to extend this first result. We shall be guided by Eq. (30) to do this. This equation establishes the factors that affect the duration $\mathrm{T}$ of the qESS. These factors are driving forces for the hectic bursts that terminate one qESS and generate a new one. The numbers and types of species in the sequences of qESS in the TaNa model may (and do) change from one qESS to the next. This occurs at the hectic bursts. If this feature is to be reproduced, the period of the attractor associated with the tangent bifurcation should have the capability to be changed. Our model considers families of chaotic attractors in the vicinity of tangent bifurcations present in low-dimensional iterated maps that display intermittency, referred to as intermittency of type I. $^{2}$ For convenience, these families can be taken from those occurring an infinite number of times in unimodal maps, as represented by the quadratic logistic map, $f_{\nu}(x)=1-\nu x^{2},-1 \leq x \leq 1,0 \leq \nu \leq 2$.

Unimodal maps share self-similar families of attractors such as chaotic attractors that consist of $2^{k}, k=0,1,2, \ldots$, bands. For given $k$, the $2^{k}$-band attractors appear for a given range of the control parameter $\nu$, with the exception of some smaller intervals where periodic attractors reappear (see Fig. 4). These intervals are control parameter windows of regular behaviour that start at a tangent bifurcation at which chaotic dynamics transforms sharply into periodic motion. The dynamics at the chaotic attractors in the vicinity of the left edge, $\nu \lesssim \nu_{\tau}$ of the window of periodic attractors, the location $\nu=\nu_{\tau}$ of the tangent bifurcation, displays intermittency, i.e., at $\nu \lesssim \nu_{\tau}$ where $\nu_{\tau}$ is the location of the tangent bifurcation. That is, the map trajectories consist of quasi-periodic motion interrupted by bursts of irregular behaviour. The iteration time duration of the quasi-periodic episodes increases as the

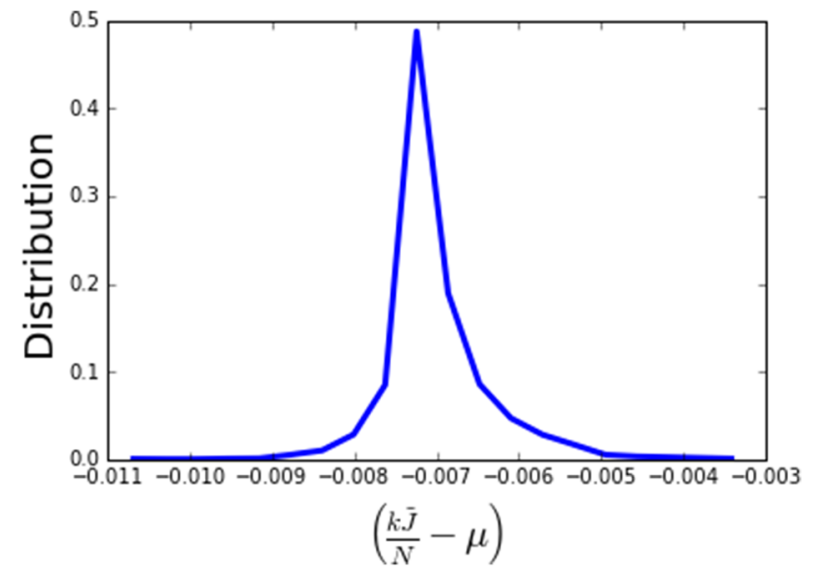

FIG. 3. The distribution of factors that enter into the expression Eq. (30) for the duration of the qESS. The $\bar{J}$ and $\tilde{J}$ are the time averaged during a qESS. The data shown are sampled over 4000 qESS realisations. The parameters for the simulation are the same as in Fig. 1. 


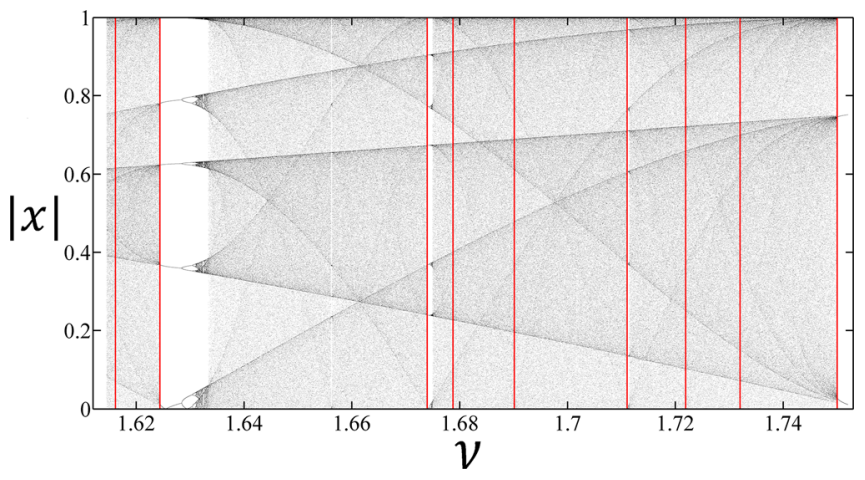

FIG. 4. Families of chaotic attractors with interspersed periodic attractor windows for the logistic map with positions in absolute values, for a range of control parameter values $\nu$. The red lines indicate the control parameter values that correspond to the segments appearing in Fig. 6. The periods associated with the vertical red lines are, from left to right, 5, 7, 12, 9, 8, 10, 11, and 3 .

tangent bifurcation is approached and the statistical features of these durations have been shown to display characteristics of various types of noise. ${ }^{4}$ At the tangent bifurcation, the duration of the episodes diverges, and the motion becomes periodic. The opening periods $\tau$ of the windows follow the Sharkovskii ordering. ${ }^{15}$ A single tangent bifurcation of a unimodal map linking a fixed period attractor to a chaotic one is only capable of portraying a fixed set of types of species and a fixed total number of them, i.e., no evolutionary change. Whereas a set of such tangent bifurcations visited sequentially according to some rules can describe consecutive quasi-stable episodes with varying numbers of species and total population, as in an evolutionary process. The following procedure, which incorporates the criteria identified above for the duration of the qESS, can be used to generate successive quasi-periodic events of different (quasi)-periods mediated by brief erratic bursts, each event associated with a different periodicity $\tau_{n}$ and of different duration $T_{n}$. First, choose a control parameter value $\nu_{0}$ just left of a window of periodicity $\tau_{0}$ of the logistic map with tangent bifurcation at $\nu_{\tau_{0}}, \delta \nu_{0} \equiv \nu_{0}-\nu_{\tau_{0}} \lesssim 0$. When the map trajectory with the initial condition $x_{0}$ comes out of the bottlenecks formed by $f^{\left(\tau_{n}\right)}(x)$ and the identity line (see Fig. 2) (to experience a chaotic burst before it is re-injected close to the bottlenecks), the control parameter of the map is changed, and the trajectory continues its evolution in a different environment. This change in the control parameter is mediated by a set of stochastic conditions, that when fulfilled, another control parameter value $\nu_{1}$ is generated just left of a window of periodicity $\tau_{1}$ with $\delta \nu_{1} \equiv \nu_{1}-\nu_{\tau_{1}} \lesssim 0$, and so on for $n=2,3, \ldots$. Two of these conditions refer to exceedances associated with two random variables $\delta_{1}$ and $\delta_{2}$, generated by a normal and a uniform distributions, respectively. The conditions are $\delta_{1}>\Gamma_{1}$ and $\delta_{2}>\Gamma_{2}$, where $\Gamma_{1}$ and $\Gamma_{2}$ are the two prescribed thresholds. Samplings for $\delta_{1}$ and $\delta_{2}$ run until the two thresholds are overcome simultaneously at a given number $l$ of times ( $l=2$ for our results shown below). Then, the control parameter value is changed to that of a different window chosen at random. The new value of $\delta \nu_{n}$ is estimated via $\delta \nu_{n} \sim\left(T_{n}\right)^{-2}$ (see, e.g., Ref. 2 Chap. 5), where the duration $T_{n}$ is given by Eq. (30). The two implemented thresholds correspond to critical values of the imbalances referred after Eq. (30)

$$
\delta_{1}=\frac{\frac{k}{N} \tilde{J}-\mu}{p_{\text {kill }}^{2}\left(1-P_{0}\right)},
$$

and

$$
\delta_{2}=\frac{\frac{k}{2 N}(\bar{J}-\tilde{J})}{p_{\text {kill }}^{2}\left(1-P_{0}\right)} .
$$

For conciseness and clarity, in Fig. 5, we show as a flow diagram the algorithm followed by the consecutive tangent bifurcation model. The repetition of this prescription leads to the dynamical behavior shown in Fig. 6 that can be compared with that obtained from the TaNa model in Fig. 1. The quasi-periodic episode of period $\tau_{n}$ is identified with the quasi stable co-existence of $n$ species for a time period $T_{n}$ in the TaNa model and the chaotic burst at its ending leads to some extinctions and new mutated species of the following quasi-stable configuration.

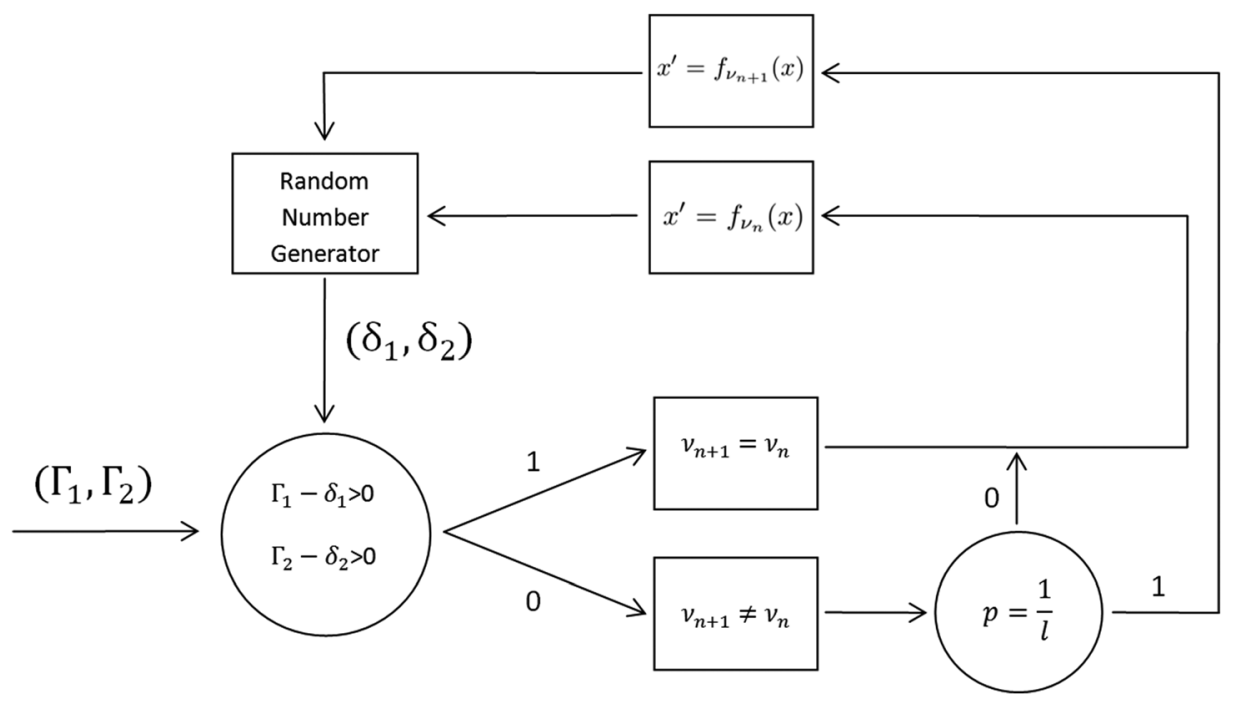

FIG. 5. Schematic flow diagram of the algorithm under which the consecutive tangent bifurcation model operates. The successive values of $\delta \nu_{n}$ are estimated via $\delta \nu_{n} \sim\left(T_{n}\right)^{-2}$. We have used $l=2$ for our numerical results. See text for description. 

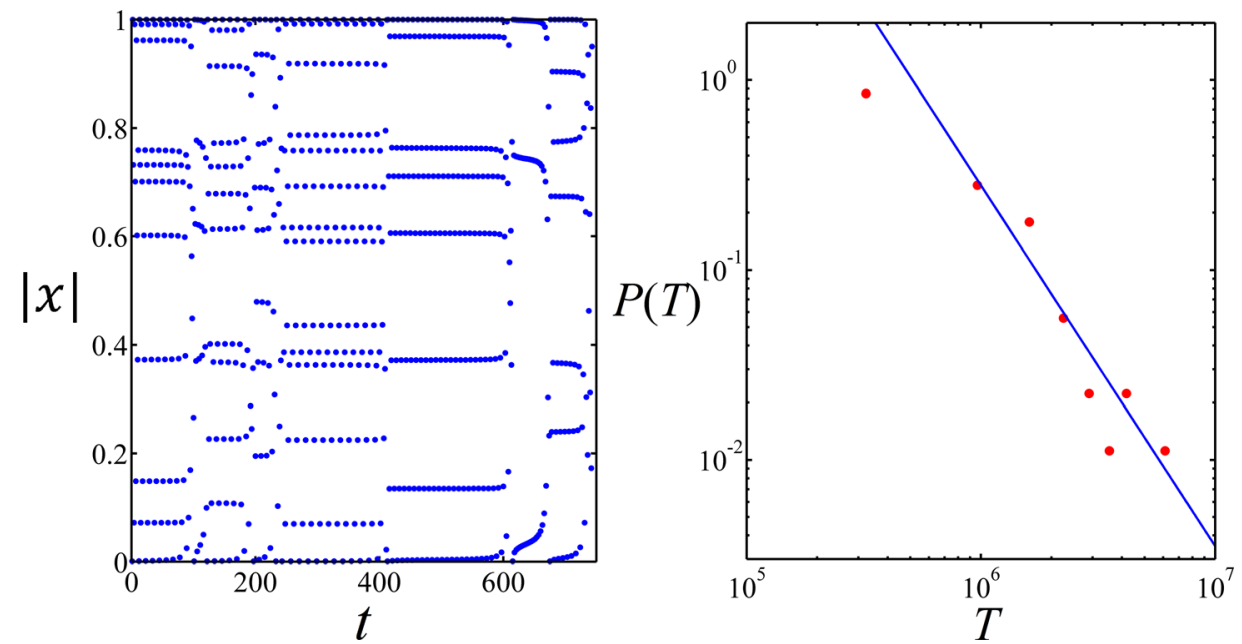

FIG. 6. Left panel: Iterated time $t$ evolution of a trajectory generated by the consecutive tangent bifurcation model. The figure is composed of segments, each of which corresponds to a fixed value of the control parameter $\nu_{n}$ close to a tangent bifurcation of a given period $\tau_{n}$. The periods of the segments are consecutively, from left to right, 11, 5, 12, 9. Positions appear in the figure in absolute values. Iteration times $t$ would correspond to generations in the TaNa model (as in left panel in Fig. 1). Right panel: Log-Log plot of the distribution of durations $T$ of the quasi stationary episodes near the tangent bifurcations of the phenomenological model. It approximates a power law with exponent -1.87 that resembles the distribution of qESS episodes obtained from simulations of the TaNa model in Ref. 6.

This approach can be considered to be a phenomenological modelling of the original TaNa model. The threshold selections of the periodic windows $\tau_{n}$ at $\nu_{\tau_{n}}$ and of the value of the control parameter distance $\delta \nu_{n}$ from the corresponding tangent bifurcation can be further elaborated, e.g., by devising specific rules suggested by ecological principles associated with reproduction, mutation, and death, and in this way obtain a closer reproduction of the dynamics of the TaNa model. Interestingly, an average decrement of the variables $\delta \nu_{n}$ with increasing time $t$, that implies an average increment of the duration of quasi-stable episodes $T_{n}$ with $t$, observed in the TaNa dynamical properties, signals an approach to the intermittency transition out of chaos. Our modelling by means of the dynamics associated with families of tangent bifurcations implies (in a well-defined manner restricted to deterministic nonlinear dynamics) that the ecological evolution model operates near the onset of chaos, in our case, at nearly vanishing Lyapunov exponent.

The stochastic element of the model resides only in the threshold conditions to switch from the chaotic vicinity of one tangent bifurcation to the chaotic vicinity of another. The dynamical behavior of the model between these instantaneous shift times is the known chaotic deterministic dynamics in the neighborhood of tangent bifurcations of low-dimensional attractors. In the model, the time duration between the control parameter $\nu$ changes corresponds to the duration of the qESS (see Fig. 6).

\section{DISCUSSION AND CONCLUSION}

To explore a possible relationship between the dynamical properties of low-dimensional nonlinear systems and the high-dimensional, and often stochastic, dynamics of relevance to complex systems, it is necessary to identify a few robust macroscopic degrees of freedom of the latter that capture the salient features of its dynamical evolution. We have performed such an analysis for a particularly high-dimensional and particularly strongly stochastic model, namely, the Tangled Nature model of evolutionary ecology. We find that despite the dramatic approximations involved in establishing a local one-dimensional map, we nevertheless obtain meaningful and interesting statements concerning the duration of the long quiescent epochs of the TaNa model.

We then went on to describe how a simple quadratic map is able to reproduce structures that qualitatively exhibit, with a high degree of similarity, the full high-dimensional stochastic model. We advanced a nonlinear dynamical system model consisting of consecutive one-dimensional chaotic attractors near tangent bifurcations that generate time evolution patterns resembling those of the TaNa model in macroscopic scales. These tangent bifurcations are chosen from the infinite families that occur at the onset the periodic windows in the logistic map according to a threshold prescription based on the previously identified mechanisms that control the duration of the basic quasi-stable event generated by the local map derived from the TaNa model. These mechanisms involve imbalances between (average) values of parameters with ecological meaning that define the TaNa model. See Eq. (30) and text below it.

The manner in which randomness has been introduced in the consecutive tangent bifurcation model does not generate any unstable behavior in the low-dimensional intermittency. The random changes in the control parameter take place instantaneously according to the algorithm described in the previous section. Otherwise, the dynamics is fully deterministic and follows the known patterns associated with the families of tangent bifurcations in a quadratic map.

The generally unanticipated link we established between the macroscopic dynamics of a high dimensional stochastic model and the intermittent dynamics of low-dimensional systems requires a closer examination. This can be developed, first, by deriving under less sweeping approximations the collapse of degrees of freedom that leads to this correspondence. This would include the derivation from the TaNa 
model of a more complete, tangent bifurcation model to fit more closely the mutual interactions that define the TaNa model of evolutionary ecology. The occurrence of the connection between high- and low-dimensional dynamical model systems offers a new path to the study of complex systems. A possible methodology to obtain, or at least to visualize, this reduction is, as attempted here, to derive from the original model via mean-field arguments a nonlinear iterated map representation, such as a coupled map lattice. This is actually the case here for the species or agents $n_{i}$ of the TaNa model when described by the approximations made on the likelihood function $H_{i}$. Such macroscopic collective behavior is known to arise in couple map lattices. ${ }^{16}$

\section{ACKNOWLEDGMENTS}

A.R. and A.D.-R. acknowledges support from DGAPAUNAM-IN103814 and CONACyT-CB-2011-167978 (Mexican Agencies). H.J.J. was supported by the European Project CONGAS (Grant No. FP7-ICT-2011-8-317672).

${ }^{1}$ P. Sibani and H. J. Jensen, Stochastic Dynamics of Complex Systems (Imperial College Press, 2013).

${ }^{2}$ H. G. Schuster and W. Just, Deterministic Chaos: An Introduction (WileyVCH Publishers, 2005).

${ }^{3} \mathrm{~B}$. Hu and J. Rudnick, "Exact solutions to the Feigenbaum renormalizationgroup equations for intermittency,” Phys. Rev. Lett. 48, 1645 (1982).
${ }^{4}$ I. Procaccia and H. G. Schuster, "Functional renormalization-group theory of universal 1/f noise in dynamical systems," Phys. Rev. A 28, 1210 (1983).

${ }^{5}$ Y. Pomeau and P. Manneville, "Intermittent transition to turbulence in dissipative dynamical systems," Commun. Math. Phys. 74, 189-197 (1980).

${ }^{6}$ K. Christensen, S. A. di Collobiano, M. Hall, and H. J. Jensen, "Tangled nature model: A model of evolutionary ecology," J. Theor. Biol. 216, 73-84 (2002).

${ }^{7}$ M. Hall, K. Christensen, S. A. di Collobiano, and H. J. Jensen, "Timedependent extinction rate and species abundance in a tangled-nature model of biological evolution,” Phys. Rev. E 66, 011904 (2002).

${ }^{8}$ P. A. Rikvold and R. K. P. Zia, "Punctuated equilibria and 1/f noise in a biological coevolution model with individual-based dynamics," Phys. Rev. E 68, 031913 (2003).

${ }^{9}$ R. K. P. Zia and P. A. Rikvold, "Fluctuations and correlations in an individual-based model of evolution,” J. Phys. A 37, 5135-5155 (2004).

${ }^{10}$ N. Becker and P. Sibani, "Evolution and non-equilibrium physics: A study of the tangled nature model," Europhys. Lett. 105, 18005 (2014).

${ }^{11}$ A. E. Nicholson and P. Sibani, "Cultural evolution as a nonstationary stochastic process," Complexity 214-223 (2015).

${ }^{12}$ P. Vazquez, J. A. del Rio, K. G. Cedano, M. Martinez, and H. J. Jensen, "An entangled model for sustainability indicators," PLoS One 10, e0135250 (2015).

${ }^{13}$ P. G. Higgs and B. Derrida, "Genetic distance and species formation in evolving populations," J. Mol. Evol. 35(5), 454-465 (1992).

${ }^{14}$ J. M. Smith, Evolution and the Theory of Games (Cambridge University Press, 1982).

${ }^{15}$ M. Schroeder, Fractals, Chaos, Power Laws: Minutes from an Infinite Paradise (Freeman, 1991).

${ }^{16} \mathrm{H}$. Chaté and P. Manneville, "Emergence of effective low-dimensional dynamics in the macroscopic behaviour of coupled map lattices," Europhys. Lett. 17, 291 (1992). 